\title{
Variable imprinting of the MEST gene in human preimplantation embryos
}

\author{
John D Huntriss ${ }^{\star}, 1$ Karen E Hemmings ${ }^{1}$, Matthew Hinkins ${ }^{1}$, Anthony J Rutherford ${ }^{2}$, Roger G Sturmey ${ }^{3}$, \\ Kay Elder ${ }^{4}$ and Helen M Picton ${ }^{1}$
}

There is evidence that expression and methylation of the imprinted paternally expressed gene 1 /mesoderm-specific transcript homologue (PEG1/MEST) gene may be affected by assisted reproductive technologies (ARTs) and infertility. In this study, we sought to assess the imprinting status of the MEST gene in a large cohort of in vitro-derived human preimplantation embryos, in order to characterise potentially adverse effects of ART and infertility on this locus in early human development. Embryonic genomic DNA from morula or blastocyst stage embryos was screened for a transcribed Aflll polymorphism in MEST and imprinting analysis was then performed in cDNA libraries derived from these embryos. In 10 heterozygous embryos, MEST expression was monoallelic in seven embryos, predominantly monoallelic in two embryos, and biallelic in one embryo. Screening of cDNA derived from 61 additional human preimplantation embryos, for which DNA for genotyping was unavailable, identified eight embryos with expression originating from both alleles (biallelic or predominantly monoallelic). In some embryos, therefore, the onset of imprinted MEST expression occurs during late preimplantation development. Variability in MEST imprinting was observed in both in vitro fertilization and intracytoplasmic sperm injection-derived embryos. Biallelic or predominantly monoallelic MEST expression was not associated with any one cause of infertility. Characterisation of the main MEST isoforms revealed that isoform 2 was detected in early development and was itself variably imprinted between embryos. To our knowledge, this report constitutes the largest expression study to date of genomic imprinting in human preimplantation embryos and reveals that for some imprinted genes, contrasting imprinting states exist between embryos.

European Journal of Human Genetics (2013) 21, 40-47; doi:10.1038/ejhg.2012.102; published online 4 July 2012

Keywords: imprinting; PEG1/MEST; assisted reproduction; imprinted; epigenetic; preimplantation

\section{INTRODUCTION}

Paternally expressed gene $1 /$ mesoderm-specific transcript homologue (PEG1/MEST) encodes an $\alpha / \beta$ hydrolase fold family enzyme of unknown function. In mice, Mest maps to an imprinted region that affects growth ${ }^{1}$ and disruption of the Mest gene leads to embryonic growth retardation and abnormal maternal nurturing behaviour. ${ }^{2}$ Mest expression is upregulated in obese adipose tissue and may regulate adipocyte growth and lipid accumulation. ${ }^{3-6}$ In humans, the imprinted MEST gene maps to chromosome $7 \mathrm{q} 32,{ }^{7,8}$ and is a candidate gene for Silver-Russell syndrome (SRS), although no corresponding sequence or epigenetic mutations in MEST have yet been reported. ${ }^{9-11}$ In mice, loss of imprinting (LOI) of Mest is associated with altered growth. ${ }^{12}$ LOI of MEST in humans has been described in colorectal cancer ${ }^{13}$ and lung-cancer cell lines. ${ }^{14}$ LOI has also been reported in invasive breast cancer, ${ }^{15}$ but the biallelic expression observed here is likely to be the result of a promoter usage switch between MEST isoforms. ${ }^{16}$

Assisted reproductive technology (ART) and infertility may be associated with epigenetic defects. ${ }^{17}$ There is a suggestive evidence that methylation and expression of the MEST gene may be affected by various forms of ART in humans, mice and non-human primates. Hypermethylation at the MEST differentially methylated region (DMR), has been reported in a girl with SRS who was conceived by in vitro fertilisation (IVF), ${ }^{18}$ although it is unclear whether this paternally inherited epimutation was responsible for the syndrome. Aberrant DNA methylation of MEST has also been observed in superovulated human oocytes. ${ }^{19}$ Differential methylation at MEST has been observed between children conceived in vitro and in vivo ${ }^{20}$ and has been observed to differ between children conceived through IVF as compared with intracytoplasmic sperm injection (ICSI) and naturally conceived children. ${ }^{21}$ In mice, the Mest methylation imprint may be susceptible to the effects of in vitro culture or ageing of oocytes in vitro. ${ }^{22}$ Significant reduction in DNA methylation at Mest has been reported in mouse oocytes generated after ovarian follicle culture under low methyl donor levels. ${ }^{23}$ There may also be adverse effects imposed on the murine Mest gene induced by in vitro folliculogenesis, leading to a loss of methylation. ${ }^{24}$ Finally, overexpression of the MEST gene has been described following in vitro maturation of rhesus monkey oocytes. ${ }^{25}$

Aberrant methylation at MEST is associated with certain forms of male infertility. Hypermethylation of MEST has been described in males with oligozoospermia, ${ }^{26,27}$ and also idiopathic male infertility. ${ }^{28}$ MEST DMR methylation errors were the most common defects observed in infertile men relative to several other imprinted genes. ${ }^{29}$ Global sperm DNA methylation analysis has also revealed that elevated methylation of MEST is associated with poor sperm parameters. ${ }^{30}$

${ }^{1}$ Division of Reproduction and Early Development, Leeds Institute of Genetics, Health and Therapeutics, University of Leeds, The LIGHT Laboratories, Leeds, UK; ${ }^{2}$ The Leeds Centre For Reproductive Medicine, Leeds Teaching Hospitals NHS Trust, Seacroft Hospital, Leeds, UK; ${ }^{3}$ Centre for Cardiovascular and Metabolic Research, Hull York Medical School, University of Hull, Hull, UK; ${ }^{4}$ Bourn Hall Clinic, Cambridge, UK

*Correspondence: Dr JD Huntriss, Division of Reproduction and Early Development, Leeds Institute of Genetics, Health and Therapeutics, University of Leeds, The LIGHT Laboratories, Clarendon Way, Leeds, West Yorkshire LS2 9JT, UK. Tel: +44 (0)113 343 7056; Fax: +44 (0)113 343 6603; E-mail: j.huntriss@leeds.ac.uk

Received 7 November 2011; revised 12 March 2012; accepted 12 April 2012; published online 4 July 2012 
Collectively, this data implies that the MEST locus may be susceptible to the effects of ART manipulation and aberrant epigenetic programming that occurs in infertility. In this study, we therefore sought to characterise the imprinting status of MEST in human preimplantation embryos derived by assisted reproduction.

\section{MATERIALS AND METHODS}

\section{Oocytes and embryos}

Human preimplantation embryos that were not selected for transfer, and which were therefore surplus to treatment needs were donated for research under informed consent by couples attending the Assisted Conception Unit (ACU) at Leeds General Infirmary. All tissues were donated under protocols that have been approved by the Leeds West Research Ethics Committee and licensed by the Human Fertilisation and Embryo Authority (HFEA). The procedures of ovarian stimulation, oocyte collection and embryo culture in Medi-Cult IVF medium (MediCult UK Ltd, Reigate, UK) and in vitro fertilisation were performed at the Leeds ACU according to published protocols. ${ }^{31}$ Donated, fresh embryos were transported at day-2 post insemination to the University of York as previously described ${ }^{32,33}$ and cultured to the blastocyst stage. Cryopreserved embryos that were surplus to treatment requirements were donated for research under informed consent by couples attending Bourn Hall Clinic, Cambridge and were thawed and cultured to the blastocyst stage in the HFEA licensed research laboratories in Leeds. All embryos were individually cultured in $4 \mu \mathrm{l}$ droplets of Earle's Balanced Salt Solution, supplemented with $1 \mathrm{~mm}$ glucose, $5 \mathrm{~mm}$ lactate, $0.47 \mathrm{~mm}$ sodium pyruvate, $0.5 \%(\mathrm{v} / \mathrm{v})$ human serum albumin (Zenalb 20; Bioproducts Lab, Elstree, UK) and amino acids at close-to-physiological concentrations, based on the data of Tay et al $1997,{ }^{34}$ under embryo tested mineral oil at $37^{\circ} \mathrm{C}$ under $5 \% \mathrm{CO}_{2}$ in air. Embryos were transferred to fresh drops of culture medium after each $24 \mathrm{~h}$ culture period. The morphological grade of each morula or blastocyst was recorded at the end of culture as described previously. ${ }^{32}$ Only embryos of grades 1-3 were used. At the end of culture, embryos were allowed to perish by cooling to room temperature over 10 minutes in ambient atmospheric conditions, washed in $\mathrm{Ca}^{2+}$ - and $\mathrm{Mg}^{2+}$-free phosphate-buffered saline at $4{ }^{\circ} \mathrm{C}$ (Life Technologies, Paisley, UK), before being snap frozen in lysis buffer (Dynabeads, Life Technologies). For embryo sample numbers 9 and 10 (Figure $2 \mathrm{~b}$ ) at the end of culture, the zona pellucidae were removed by exposure to Acid Tyrode's solution (Sigma-Aldrich, Gillingham, UK).

\section{Reverse transcription and cDNA amplification}

Single embryos and oocytes were collected and lysed at $80^{\circ} \mathrm{C}$ in lysis buffer (Dynabeads mRNA DIRECT Micro Kit, Life Technologies). Following RNA extraction with oligo-dT Dynabeads, cDNA was generated and amplified using an adaptation of existing cDNA amplification protocols ${ }^{35,36}$ using $1 \mu \mathrm{g}$ each of the cDNA amplification primers, primer 1: $5^{\prime}$-AAACGACGGCCAGTGAATTG TAATACGACTCACTATAGGGCGCT $24^{-} 3^{\prime}$ and primer 2: $5^{\prime}$-AAGCAGTGGTAT CAACGCAGAGTACGCGGG-3' using superscript II RNAseH-reverse transcriptase (Invitrogen) and associated reagents with incubation for $2 \mathrm{~h}$ at $42^{\circ} \mathrm{C}$. The cDNA was amplified by PCR using an additional $1 \mu \mathrm{g}$ of each primer, $2 \mu \mathrm{l}$ $50 \times$ advantage 2 polymerase (Clontech, Mountain View, CA, USA), in a thermal cycler for 32 cycles of $95^{\circ} \mathrm{C}$ for $45 \mathrm{~s}, 65^{\circ} \mathrm{C}$ for $6 \mathrm{~min} 45 \mathrm{~s}$. The embryonic cDNA libraries are used across multiple experiments.

\section{Genotyping embryos}

Genomic DNA was extracted from embryos using the Qiagen DNA Micro kit (Crawley, UK) according to protocol, utilising the cell lysate that was residual from the mRNA extraction process. Two rounds of PCR were performed on $2.5 \mu \mathrm{l}$ of extracted genomic DNA using the primer PEG1-gDNA-F GGTCCTGGCCATCAAACATA $^{37}$ and R1 $5^{\prime}$-CCTCTACTGTAGCTCAAGA G- $3^{\prime 38}$ in the first round, and PEG1-gDNA-F and R2 $5^{\prime}$-GGAGTCTCAGACT GTTATTTGC- $3^{\prime 38}$ in the second round (Figure 1a). PCR conditions were $94^{\circ} \mathrm{C}$ for $5 \mathrm{~min}$, then $35 \mathrm{cycles}$ of $94^{\circ} \mathrm{C}$ for $30 \mathrm{~s}, 60^{\circ} \mathrm{C}$ for $30 \mathrm{~s}, 72^{\circ} \mathrm{C}$ for $1 \mathrm{~min} 30 \mathrm{~s}$. Second round PCR products were split and one-half treated with three units of AflIII and digested at $37^{\circ} \mathrm{C}$ overnight, followed by a subsequent addition of two further units AflIII for a further $3 \mathrm{~h}$ to completion. Products were run on a $1.5 \%$ agarose gel with reference to $100 \mathrm{bp}$ ladder and enzyme digestion controls. Alternatively, embryos were genotyped by sequencing of MEST genomic PCR products (Biomolecular Analysis Facility, University of Leeds, Leeds, UK).

\section{Imprinting analysis and isoform 2-specific PCR}

For heterozygous (genotyped) embryos and the screening cDNAs of embryos of unknown genotype, the expressed alleles of the MEST gene were analysed by PCR from $50 \mathrm{ng}$ amplified embryonic cDNA using the MEST primers F1

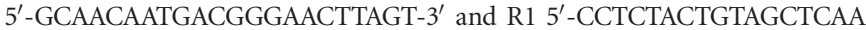
GAG- $3^{\prime}$ in the first round, and F2 $5^{\prime}$-TCAGAGGAAGAAGTTCAGAAGG- $3^{\prime}$ and R2 $5^{\prime}$-GGAGTCTCAGACTGTTATTTGC- $3^{\prime}$ in the second round. ${ }^{38}$ Half of the PCR product was digested with AflIII as described above. Appropriate enzyme digestion controls that were amplified from limiting quantities of somatic tissue mRNA were run in each experiment. Alternatively, MEST PCR products were sequenced. For isoform-specific PCR (not spanning the AflIII polymorphism), exon A (5'-CCTGTAGGCAAGGTCTTACCTG-3') and exon 1 $\left(5^{\prime}\right.$-GCGGCGGGCGGCATGGGATA-3') specific primers $^{16}$ were used in conjunction with EXON 2 R1 $5^{\prime}$-GAAGACTTCCATGAGTGAAGGGC- $3^{\prime}$ in the first round and the forward primers were then hemi-nested with EXON 2 R2 $5^{\prime}$-ATGTGCAGGTACGCAGCAAG- $3^{\prime}$ in the second round. For isoform 2-specific PCR spanning the AflIII polymorphism, Exon A and R1 were used in the first round, with primer F1 and primer R2 in second. ${ }^{38}$ RT-PCR product identity from selected embryos was confirmed by direct sequencing using primers F1 and R1. Allele ratios were quantified using the average of three peak readings per band using Image J (http://rsbweb.nih.gov/ij/). Predominantly monoallelic expression was defined as an allele ratio greater than 1:3, as described previously. ${ }^{39,40}$ PCR products obtained using isoform-specific primers were sequenced by cloning into TOPO-TA (Invitrogen) using standard M13 primers. Other primers described here are: GAPDH forward 5'-TTGTCAAGCTCATTTCCTGGTAT-3', GAPDH reverse 5'-TCTCTCTTCCT CTTGTGCTCTTG-3' ${ }^{\prime}$ ZP2 forward 5'-GACCTGCCCTGTGTCCTCTA-3' ${ }^{\prime}$, ZP2 reverse 5' -AGATCAGATGAGCCGACAC-3', ZP3 forward 5'-GATATACATCAC CTGCCACC-3', ZP3 reverse 5' -TCACTTCATGGTCACCACTC-3'.

\section{RESULTS}

\section{Embryonic genomic DNA and cDNA screening}

A nested PCR around the AflIII polymorphism in the $3^{\prime}$-untranslated region of the MEST gene was used for the purpose of genotyping embryos and imprinting analysis (Figure 1a). For the present study, parental DNA samples were unavailable for genotyping and thus we were unable to identify informative heterozygous embryos in this way. Therefore, we developed a technique that allows extraction of embryonic genomic DNA from the residual wash buffer that is remaining after the mRNA isolation procedure, to allow direct genotyping of the embryonic DNA (Figure 1b). While sperm may remain adhered to the zona on IVF-derived stage embryos, the observations from this study suggest that sperm DNA was degraded by the morula or blastocyst stage and did not therefore amplify in the genomic screening PCRs. Furthermore, ICSI embryos (no adherent sperm) and zona-stripped embryos were also included in the study. Details regarding all embryos used in the study are presented in Table 1 . We identified 10 late preimplantation stage embryos that were heterozygous for the AflIII polymorphism in the MEST gene.

\section{MEST imprinting analysis in heterozygous embryos}

For MEST imprinting analysis, cDNA libraries that were generated from human preimplantation embryos were validated by screening for transcripts of housekeeping genes and appropriate developmental marker genes including $\beta$-actin (ACTB), GAPDH, HPRT and OCT4 (Figure 1c). Allelic expression was assessed in the 10 heterozygous embryos using a nested-PCR scheme that amplifies all known MEST isoforms. In Figure 2a, PCR amplification of MEST transcripts was performed from the cDNA of five of the heterozygous embryos. 
PCR products were digested with AflIII alongside appropriate enzyme digestion controls derived from cDNA libraries that were amplified from limiting quantities of somatic tissue mRNA $(0.5 \mu \mathrm{g})$, which were either homozygous for each allele (A or G) or heterozygous. Allele ratios for all embryos are shown in Table 1. Imprinting analysis for sample 1, a grade-2, day-6 IVF morula demonstrated predominantly monoallelic expression of MEST (Figure 2a, sample 1) with $93 \%$ to $7 \%$ expression of the $\mathrm{A}$ and $\mathrm{G}$ alleles, respectively. Thus, there was weak expression detectable from the minor allele (the inferred maternal allele). In contrast, in four further late preimplantation stage embryos, expression was strictly monoallelic (Figure 2a, samples 2-5). These samples included a grade-2 IVF blastocyst (sample 2), a grade-2, day-7 IVF blastocyst (sample 3), a grade-2, day-7 IVF blastocyst (sample 4) and a grade-2, day-6 ICSI morula (sample 5), respectively. MEST imprinting analysis was also performed in a second set of heterozygous embryos, where the embryonic genomic DNA was genotyped by sequencing the AflIII polymorphism (Figure 2b, sample numbers from 6 to 10). MEST imprinting analysis was performed in their corresponding cDNAs by sequencing and/or AflIII restriction digest. For sample 6 (a day-6 expanded ICSI blastocyst), expression was biallelic. Monoallelic MEST expression was observed in three additional blastocysts (Figure 2b, samples 7-9). Predominantly monoallelic expression was observed in a day-6 expanded IVF blastocyst (sample 10)
MEST imprinting in blastocyst and morula cDNA libraries

The observation of variable MEST imprinting in the heterozygous embryos prompted a more extensive screening of embryonic MEST imprinting using a large series of cDNA libraries $(n=61)$ derived from human blastocysts and a smaller number of morulae (Figure 3). Genomic DNA was not available for these samples. As the genotype of these embryos is unknown, these experiments are informative only if expression from both alleles is detected. However, assessing a large cohort of embryos may yield useful information about the variability of imprinting between embryos. Eight embryos were identified with expression detectable from both alleles (Figure 3). Relaxed imprinting was observed in IVF-derived embryos (Figure 3, samples 11, 12, 29, 57 and 58) and ICSI-derived embryos (samples 10, 39 and 41). Two sibling ICSI embryos obtained following treatment for male factor infertility exhibited biallelic and predominantly monoallelic expression (Figure 3, samples 39 and 41, respectively). MEST imprinting was discordant between two sibling IVF embryos that exhibited predominantly monoallelic expression and monoallelic expression (Figure 3, sample 11 and Figure 2a, sample 2, respectively).

\section{Expression of MEST isoforms in human oocytes and} preimplantation embryos

Two main isoforms of MEST are expressed in humans, isoform 1 and isoform 2, that arise from promoters $\mathrm{P} 2$ and $\mathrm{P} 1$, respectively. ${ }^{41}$ a

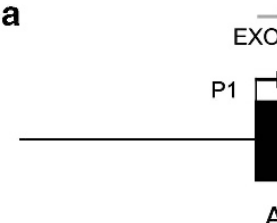

(1a)
$\overrightarrow{E X O N A}$

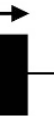

A

$(1 \mathrm{~b})$

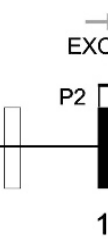

(1b) (1c)

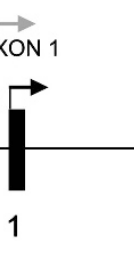

\section{b}

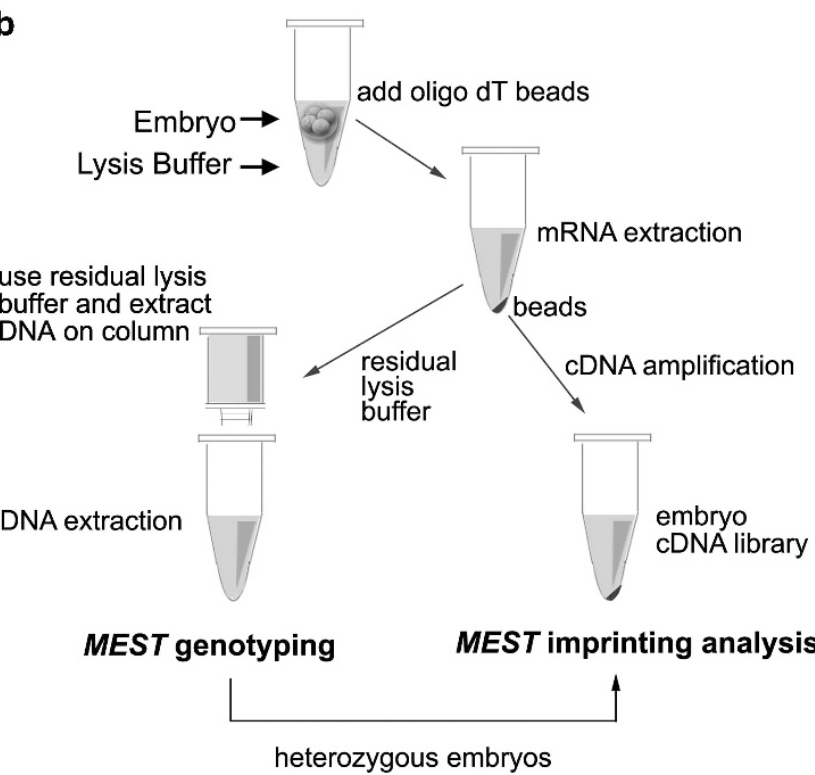

c
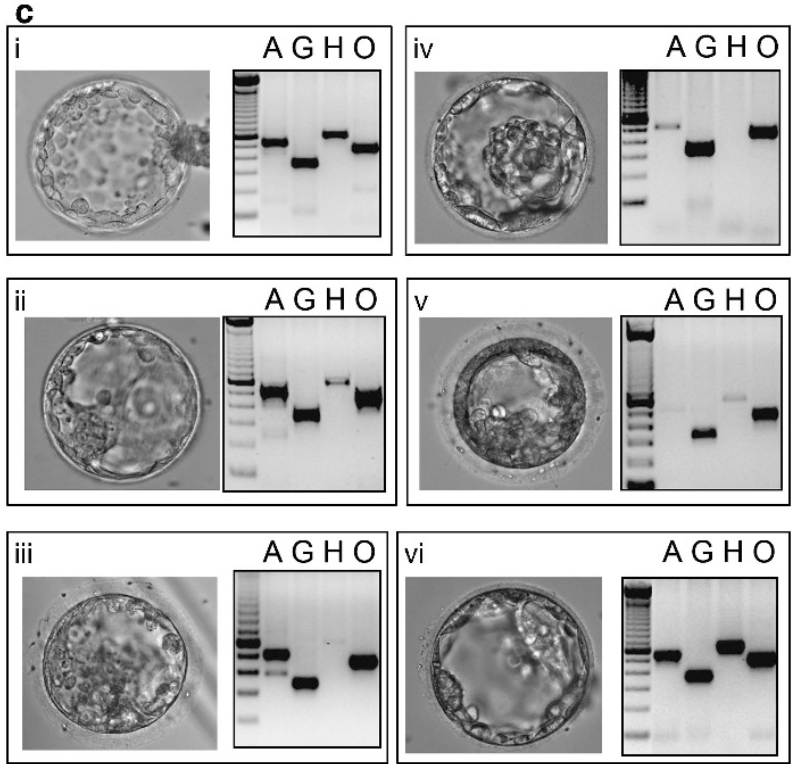

Figure 1 (a) Scheme for MEST genomic and imprinting analysis PCR assays showing PCR primer locations, PCR products and the Aflll polymorphism. (b) Scheme of protocol for the isolation of genomic DNA and cDNA from single preimplantation embryos, genotyping and imprinting analysis. (c) Examples of quality screening by PCR for housekeeping genes and developmental markers in embryonic cDNA libraries derived from six embryos (i-vi) that were used in the MEST imprinting study. Embryonic cDNAs were screened with $\beta$-actin (ACTB) gene (A), GAPDH (G), HPRT (H-variable between embryos), and OCT4 (0). 
a

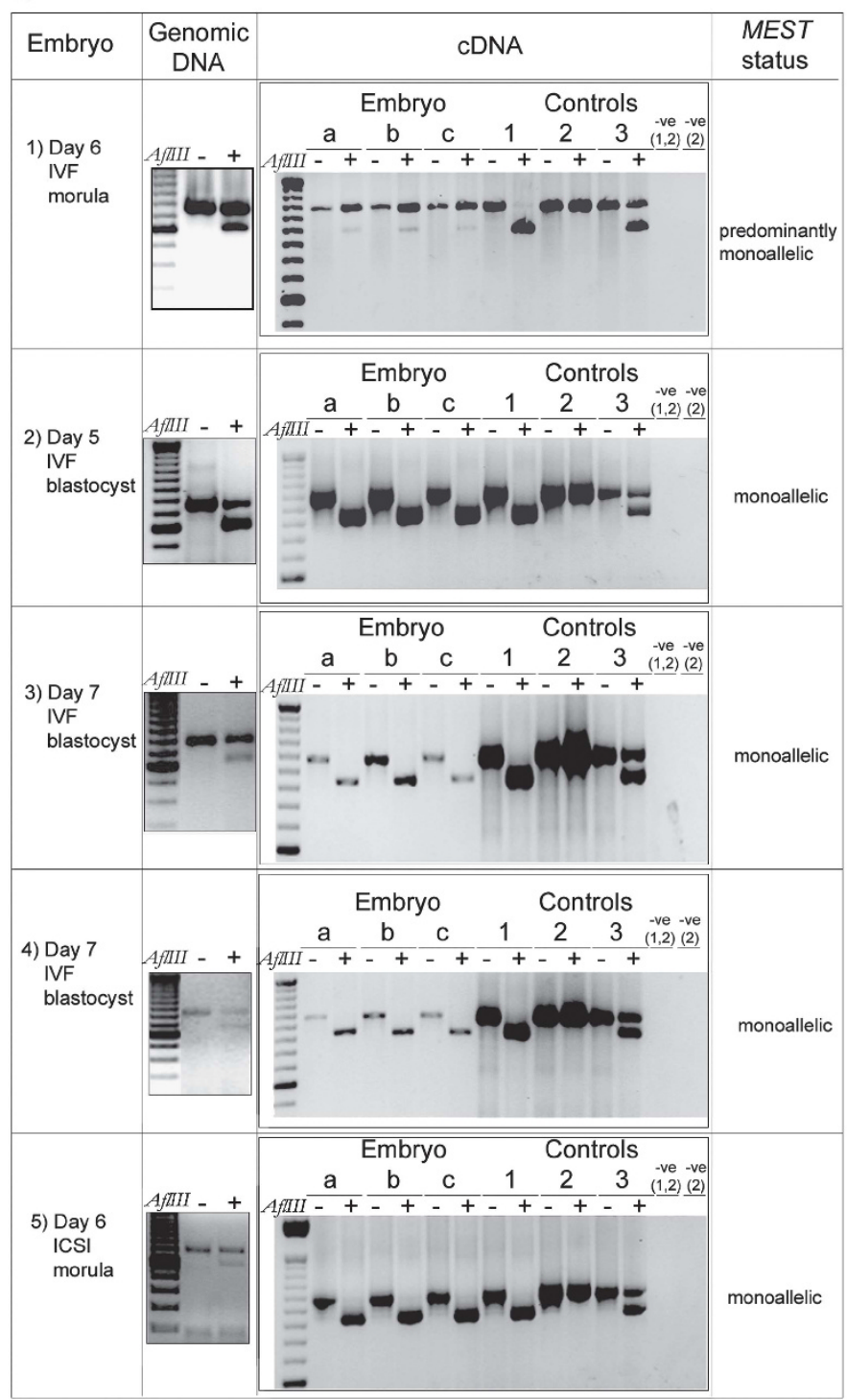

b

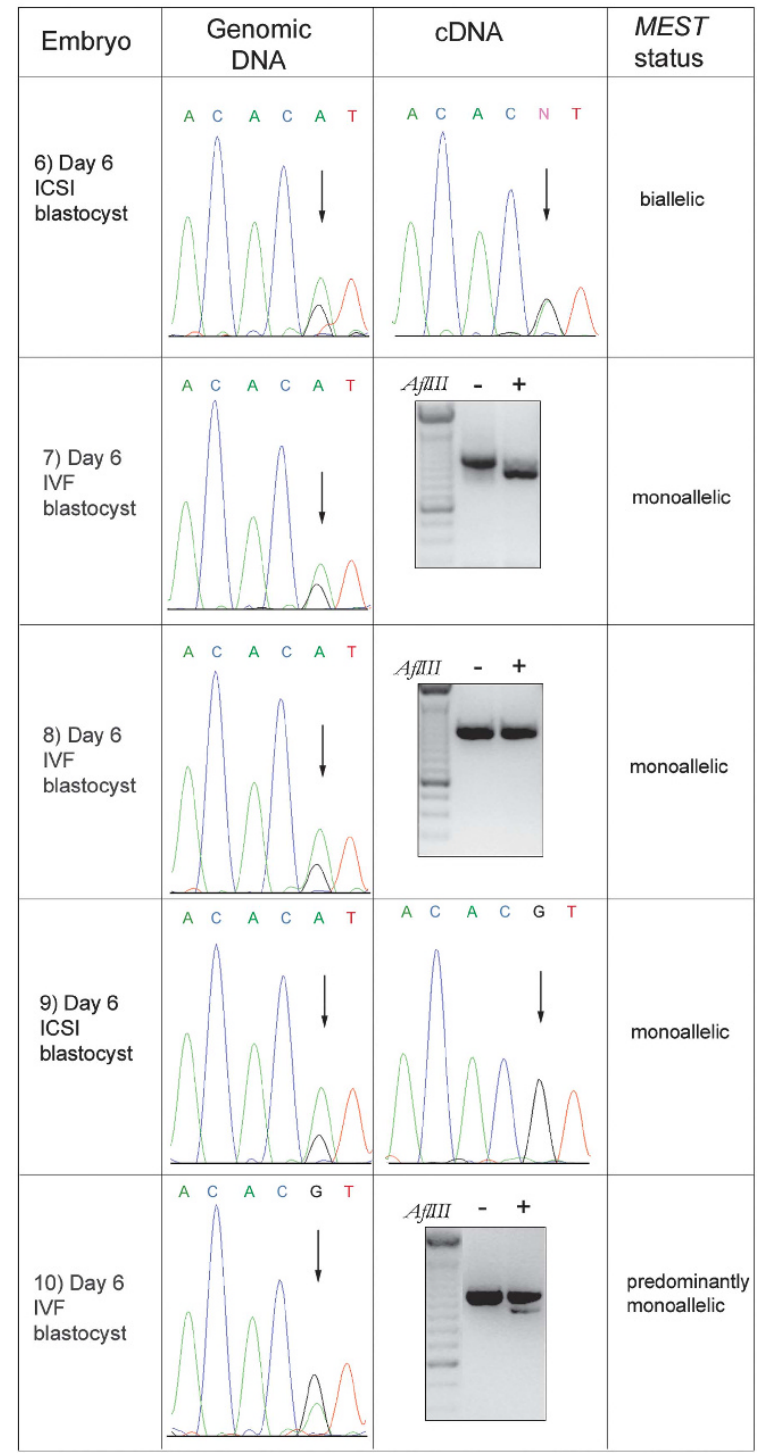

c

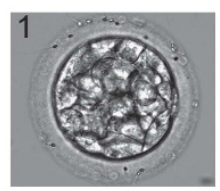

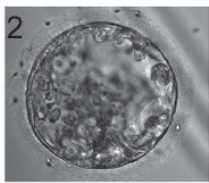

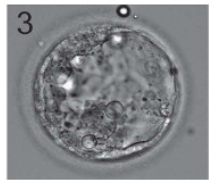

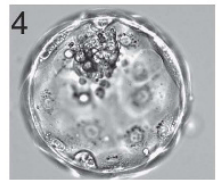

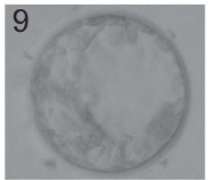

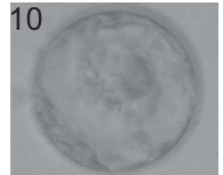

Figure 2 MEST imprinting analysis in 10 IVF and ICSI human preimplantation embryos that were heterozygous for the MEST AfIII polymorphism. The PCR scheme used for imprinting analysis from cDNA amplifies both MEST isoforms. Full sample details are given in Table 1. (a) MEST imprinting analysis by Aflll digestion in heterozygous blastocysts. Embryos were genotyped and then assessed by imprinting analysis. Nested genomic PCR and AfllI digestion of the second round PCR product was performed to identify heterozygous embryos (genomic DNA panels). Amplification of PEG1/MEST transcripts from amplified cDNA derived from each of these embryos was performed in triplicate $(a-c)$ with undigested $(-)$ and fully digested products $(+)$ in adjacent lanes (cDNA panels). For each embryo, enzyme digestion controls of MEST PCR products were included from amplified cDNA libraries derived from limiting quantities of somatic tissue RNA of known genotype. These were either homozygous for each opposite allele (control lanes 1 and 2 ) or biallelic (control lane 3). The negative sample controls include a sample carried through both rounds of PCR $(-v e 1,2)$ and the second round only $(-$ ve, 2$)$. MEST imprinting in one morula (embryo 1) was predominantly monoallelic, while monoallelic MEST expression was observed for embryos 2-5. (b) MEST imprinting analysis in an additional set of heterozygous blastocysts achieved by sequencing of MEST PCR products from the genomic DNA of five human blastocysts and analysis of MEST imprinting in their corresponding cDNAs by sequencing or Aflll restriction digest. In one embryo (embryo 6) expression was biallelic (the peaks are overlapping in the cDNA sequence), while predominantly monoallelic expression was observed in a day- 6 expanded IVF blastocyst (embryo 10). Monoallelic expression was observed in the three additional blastocysts $(7,8,9)$. (c) Photographs of selected embryos used for imprinting analysis in this figure (embryos 1, 2, 3, 4, 9, 10).

We characterised the expression of MEST isoforms in human oocytes, and preimplantation embryos. In our assay, that used a hemi-nestedPCR protocol with isoform 1-specific primers spanning from exon 1 (also referred to as 1c) to exon 2, isoform 1 was not readily detected in oocytes, zygotes or morulae samples (Figures $4 \mathrm{a}-\mathrm{c}$ ). Expression of isoform 1 was weakly detected in only one out of a total of nine 
Table 1 Summary of MEST imprinting data in 18 human preimplantation embryos. Descriptions of embryo stage and grade are given together with relative expression of each MEST allele, given as a percentage of total expression

\begin{tabular}{|c|c|c|c|c|c|c|c|c|c|}
\hline Figure & $\begin{array}{c}\text { Sample/lane } \\
\text { number }\end{array}$ & Description & Grade & $\begin{array}{l}\text { Embryo } \\
\text { status }\end{array}$ & $\begin{array}{l}\text { Cause of } \\
\text { infertility }\end{array}$ & $\begin{array}{c}\text { ART } \\
\text { treatment }\end{array}$ & MEST imprinting & $\begin{array}{c}\% \text { of } \\
\text { A allele }\end{array}$ & $\begin{array}{c}\% \text { of } \\
\text { G allele }\end{array}$ \\
\hline $2 a$ & 1 & Morula, day 6 & 2 & Fresh & Idiopathic & IVF & Predominantly monoallelic & 93.0 & 7.0 \\
\hline $2 a$ & 2 & Blastocyst & 2 & Fresh & Idiopathic & IVF & Monoallelic & 0 & 100 \\
\hline $2 a$ & 3 & Blastocyst, day 7 & 2 & Fresh & PCO & IVF & Monoallelic & 0 & 100 \\
\hline $2 a$ & 4 & Blastocyst day 7 & 2 & Fresh & PCO & IVF & Monoallelic & 0 & 100 \\
\hline $2 a$ & 5 & Morula, day 6 & 2 & Fresh & Male factor & ICSI & Monoallelic & 0 & 100 \\
\hline $2 b$ & 6 & Expanded blastocyst, day 6 & 1 & Fresh & Male factor + PCO & $|C S|$ & Biallelic & $50^{a}$ & $50^{\mathrm{a}}$ \\
\hline $2 b$ & 7 & Expanded blastocyst, day 6 & ND & Fresh & Idiopathic & IVF & Monoallelic & 0 & 100 \\
\hline $2 b$ & 8 & Expanded blastocyst, day 6 & 2.5 & Fresh & Idiopathic & IVF & Monoallelic & 100 & 0 \\
\hline $2 b$ & $9(Z S)$ & Expanded blastocyst, day 6 & ND & Frozen & Anovulation & ICSI & Monoallelic & 0 & 100 \\
\hline $2 b$ & $10(Z S)$ & Expanded blastocyst, day 6 & 1 & Frozen & Tubal + PCO & IVF & Predominantly monoallelic & 87.5 & 12.5 \\
\hline 3 & 10 & Blastocyst & 2 & Fresh & Male factor + PCO & $|C S|$ & Predominantly monoallelic & 93.9 & 6.1 \\
\hline 3 & 11 & Blastocyst & 2 & Fresh & Idiopathic & IVF & Predominantly monoallelic & 90.3 & 9.7 \\
\hline 3 & 12 & Blastocyst & 2 & Fresh & Idiopathic & IVF & Biallelic & 47.5 & 52.5 \\
\hline 3 & 29 & Blastocyst, day 7 & 2 & Fresh & Idiopathic & IVF & Predominantly monoallelic & 13.1 & 86.9 \\
\hline 3 & 39 & Blastocyst, day 6 & 1 & Fresh & Male factor + tubal & ICSI & Biallelic & 54.1 & 45.9 \\
\hline 3 & 41 & Hatching blastocyst, day 6 & 1 & Fresh & Male factor + tubal & ICSI & Predominantly monoallelic & 80.1 & 19.9 \\
\hline 3 & 57 & Early blastocyst, day 6 & ND & Fresh & Idiopathic & IVF & Predominantly monoallelic & 69.7 & 30.3 \\
\hline 3 & 58 & Blastocyst, day 6 & ND & Fresh & Idiopathic & IVF & Biallelic & 55.8 & 44.2 \\
\hline
\end{tabular}

Abbreviations: ART, assisted reproductive technologies; ICSI, intracytoplasmic sperm injection; IVF, In vitro fertilisation; MEST, mesoderm-specific transcript homologue; ND, no data; PCO, Polycystic ovaries; ZS, zona stripped.

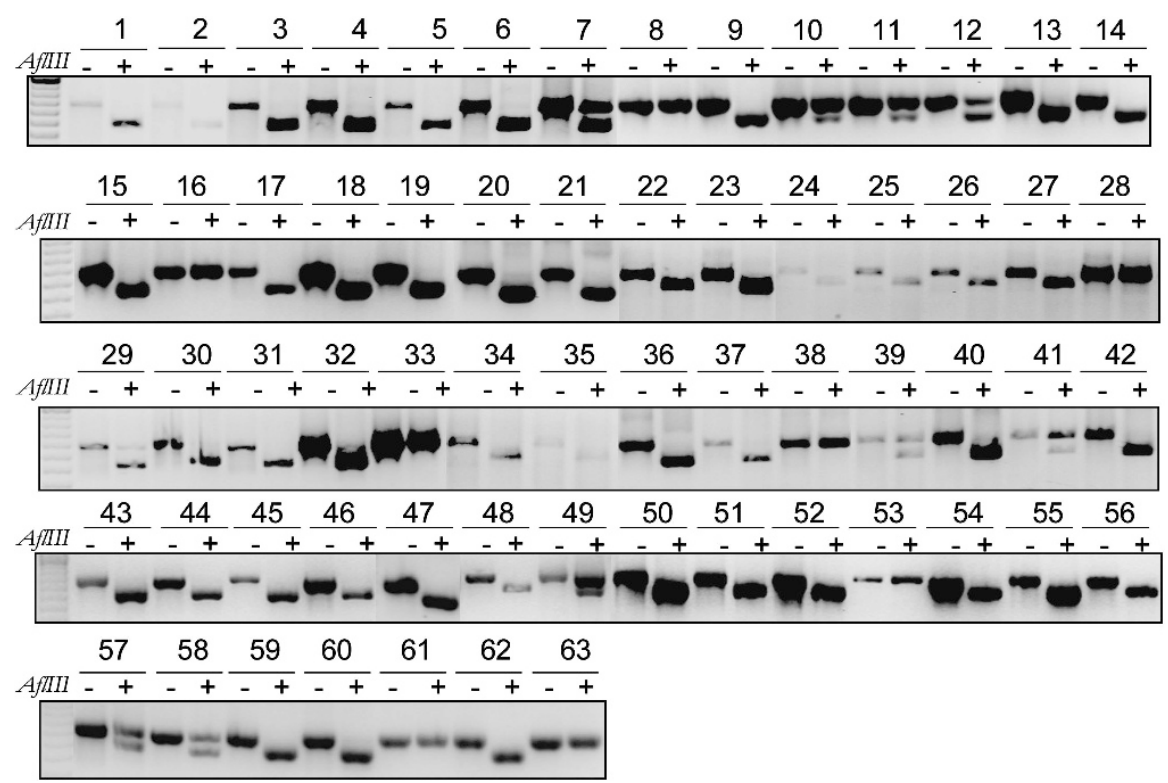

Figure 3 MEST imprinting analysis in 61 human preimplantation embryos of unknown genotype. The PCR assay amplifies both MEST isoforms. Undigested $(-)$ and fully Aflll digested products $(+)$ are shown in adjacent lanes. Control biallelic or predominantly monoallelic samples that were derived from mixed somatic tissues and liver are included (sample 7 and 49, respectively). Both MEST alleles were detected in embryo samples 10, 11, 12, 29, 39, 41, 57, 58. Embryo details and MEST imprinting status are summarised in Table 1. In this figure, Sample 39 and 41 are sibling ICSI-derived embryos. Sample 11 (Figure 3 ) is a sibling of sample 2 in Figure 2a, both are IVF-derived.

blastocysts, but was readily detected in control cDNAs derived from mixed human tissue, ovary and placenta (Figure 4c). In contrast, isoform 2 was detected in oocytes, zygotes, morulae and blastocyst stage embryos in addition to the control tissues using isoform 2-specific primers spanning from exon A (also referred to as 1a) to exon 2 (Figures $4 \mathrm{a}-\mathrm{c}$ ). The identity of the isoform-specific PCR products was confirmed by sequencing. MEST isoform 2 is the predominant MEST isoform that we detected in human oocytes and preimplantation embryos.

\section{Alternative splicing of isoform 2}

Alternative splicing within exon A of isoform 2 has been previously reported. ${ }^{42}$ The shorter Pla transcript of isoform 2 was the major splice variant expressed in human oocytes, zygotes and all preimplantation stages tested here (Figure $4 \mathrm{~b}$ ). The larger P1b transcript was detected as a weaker band in two oocytes. The identity of PCR products was confirmed by sequencing.

Direct imprinting analysis of isoform 2 in morula and blastocyst stage embryos

Isoform 2-specific imprinting analysis was performed in three embryos (Figure 4d), to ascertain whether the variable MEST imprinting observed between embryos was attributable to variable imprinting of isoform 2. In Figure 4d, sample 1 was an ICSI blastocyst that had been previously been shown to have predominantly monoallelic MEST expression when analysed by the PCR assay that 
a

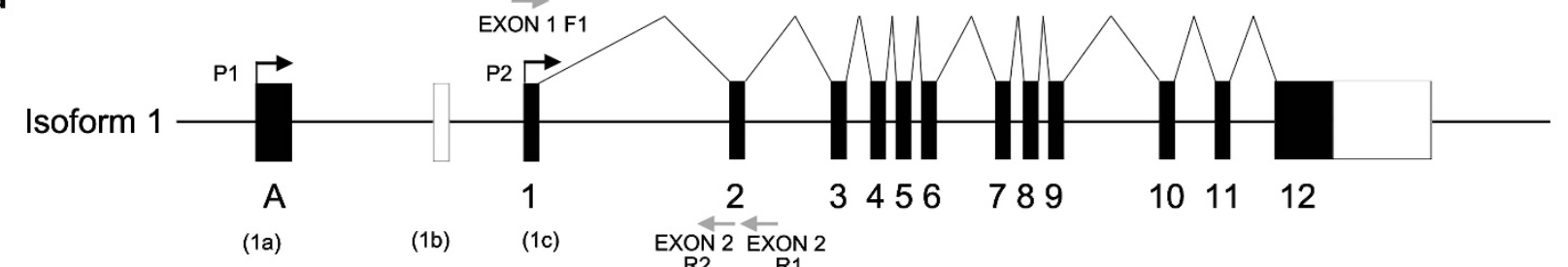

(1a)

(1b)

(1c) $\underset{\mathrm{R} 2}{\operatorname{EXON} 2} \underset{\mathrm{R} 1}{\text { EXON } 2}$

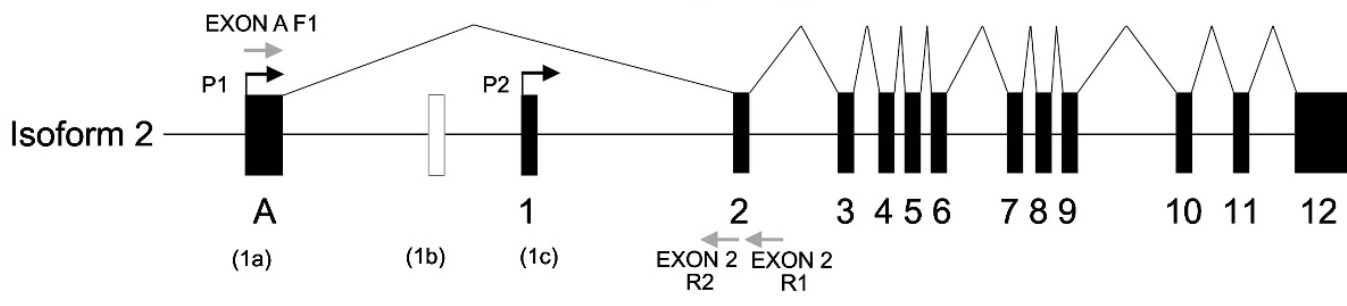

b

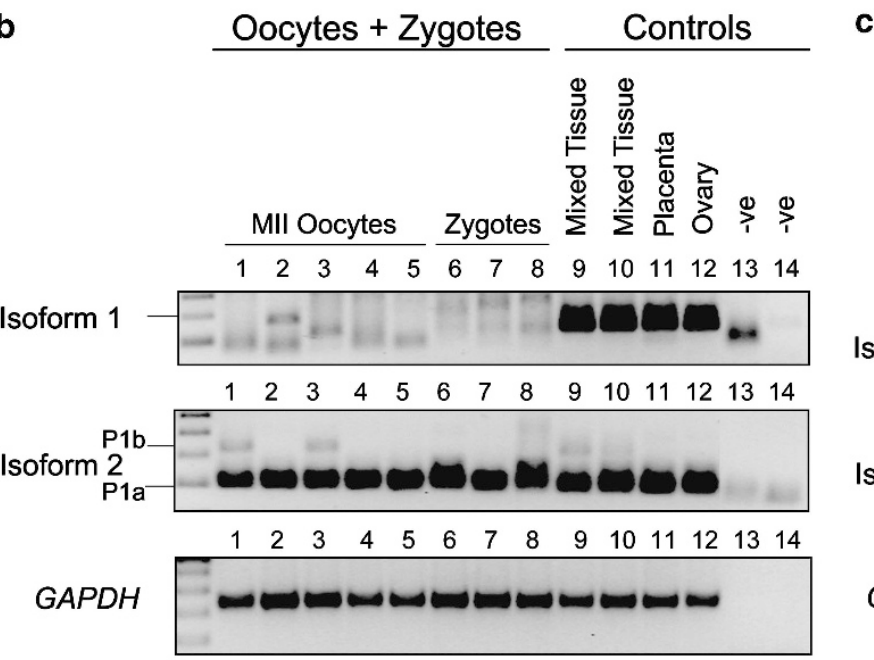

c

\section{Preimplantation Embryos Controls}
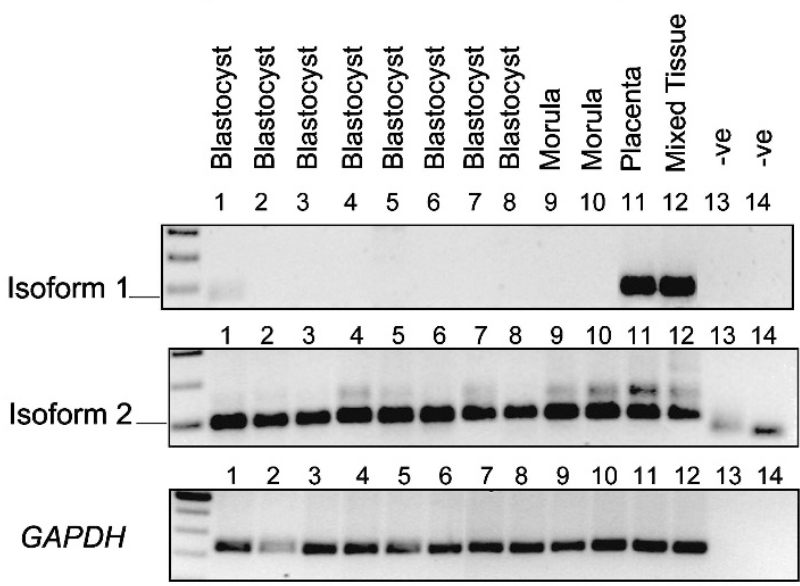

d

e
Isoform 2-specific imprinting analysis

\begin{tabular}{|c|c|c|c|c|c|c|c|c|}
\hline \multirow[b]{2}{*}{ AfIIII } & & & \multicolumn{2}{|c|}{2} & \multicolumn{2}{|c|}{3} & 4 & 5 \\
\hline & - & + & - & + & $=$ & $t$ & -ve & -ve \\
\hline 2 & & & & $=$ & & & & \\
\hline
\end{tabular}

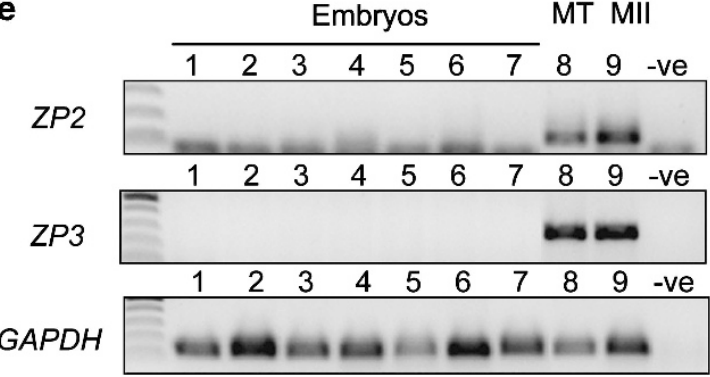

Figure 4 Expression of MEST isoforms in human oocytes, zygotes and preimplantation embryos. (a) Expression of isoform 1 was assessed using a heminested-PCR protocol with isoform 1-specific primers spanning from exon 1 (also referred to as 1c) to exon 2. Expression of isoform 2 was detected using primers spanning from exon A (also referred to as 1a) through to exon 2. (b) Expression of Isoforms 1 and 2 in human oocytes (lanes 1-5) and zygotes (lanes 6-8). Isoform 1 was weakly detected in only one oocyte (Isoform 1, lane 2) but was detected in control samples comprising of mixed somatic tissues (Isoform 1 experiment, lanes 9 and 10), and placenta and ovary (lanes 11 and 12) in the same assay. Isoform 2 was detected in all cDNAs from oocytes and zygotes (Isoform 2 experiment, lanes 1-8) and controls (lanes 9-12). Isoform 2 may be alternatively spliced: the shorter P1a transcript of isoform 2 was the major splice variant expressed in the oocyte, zygote and all preimplantation stages. The larger P1b transcript was only detected as a weaker band in two oocytes as indicated (Isoform 2 experiment, lanes 1 and 3). (c) Expression of Isoforms 1 and 2 in human morulae and blastocysts. Isoform 1 was weakly detected in one blastocyst (Isoform 1, lane 1, as indicated). In contrast, isoform 2 was readily detected in all morula or blastocyst stage embryos (lanes 1 to 10) and control tissues (lanes 11, 12). (d) Isoform 2-specific imprinting analysis in three embryos using first round PCR primers located in exon A and the $3^{\prime}$-UTR (R1) that select for isoform 2, and second round primers in exon 10 and $3^{\prime}$-UTR (R2) that surround the AfllI polymorphism. Sample 1: ICSI blastocyst that had been previously been shown to have predominantly monoallelic expression in the PCR assay that amplifies both isoforms (see Figure 3, sample 10), sample 2: day-6 IVF morula that had been previously been shown to have predominantly monoallelic MEST expression (see Figure 2a, sample 1), sample 3: day-6 ICSI morula, that was previously confirmed to have monoallelic MEST expression (see Figure 2a, sample 5). Lanes 4 and 5 are negative controls for the first and second round PCRs, respectively. (e) Expression of maternal (oocyte-derived) zona pellucida gene transcripts (ZP2, ZP3) and GAPDH in embryos with biallelic or predominantly monoallelic MEST expression. (Lanes 1-7) Embryos with biallelic or predominantly monallelic MEST expression. (Lane 8) Mixed human somatic tissue control (MT), (Lane 9) Pooled MII oocyte control cDNA samples (MII), -ve control ( -ve). 
amplifies both isoforms (see Figure 3, sample 10). Sample 2 was a heterozygous day-6 IVF morula that had been previously been shown to have predominantly monoallelic expression (see Figure 2a, sample 1). Sample 3 was a day-6 ICSI morula that was previously confirmed as being heterozygous and imprinted (see Figure 2a, sample 5). The results confirm variability of imprinting for isoform 2 between embryos. The lack of detection of maternal transcripts $(Z P 2, Z P 3)$ in the blastocysts that were identified to have monoallelic or biallelic MEST expression (Figure 4e) indicates that oocyte-derived transcripts are likely to be absent in these blastocysts. Therefore, MEST transcripts amplified in our assays are likely to be embryonic in origin.

\section{DISCUSSION}

The assessment of the imprinting status of imprinted genes in the human preimplantation embryo can reveal how imprinted gene regulation may be affected by ART and infertility, and potentially, how can this be avoided. These data also provide control information against which the origins of epigenetic instability of human-embryonic stem cells can be better understood. In addition to analysing epigenetic marks such as DNA methylation in early development, it is also pertinent to study the expression of imprinted genes, as their expression status is governed by the collective influence of these epigenetic marks, irrespective of their nature. To date, the imprinting status of only two genes has been established for human preimplantation development revealing that IGF2 and SNRPN are imprinted. ${ }^{43,44}$

MEST gene transcripts are known to be expressed in human oocytes and preimplantation embryos. ${ }^{38}$ However, earlier attempts at characterising the imprinting status of MEST in human cleavage stage embryos were inconclusive (described in Monk and Salpekar $\left.(2001)^{45}\right)$. In the current study, we have shown that in a large cohort of human preimplantation embryos that were mostly of the blastocyst stage, imprinting of the MEST gene was variable. Importantly, our data shows that the onset of imprinted MEST expression can indeed occur during late preimplantation development and this is consistent with the observation of a significant reduction of MEST expression at the blastocyst stage in human parthenogenetic preimplantation embryos. ${ }^{46}$

Across our whole study, we observed a total of 11 embryos in which transcripts from both alleles were detected, being biallelic (four embryos) or predominantly monoallelic (seven embryos). Indeed it is possible therefore that some aspects of the ART treatment or infertility per se may have influenced MEST imprinting in these cases. Most of the reports of disturbance of MEST imprinting in male factor infertility refer to hypermethylation of the paternal (expressed) MEST allele. $^{18,26,27}$ In the preimplantation embryo, it is unlikely that this abnormal methylation state would directly cause relaxed imprinting, namely, the onset of transcription from the normally silenced maternal allele. Therefore, the cause of relaxed MEST imprinting as observed in these embryos is likely to be maternal or embryonic in origin, such as a failure to establish correct imprinting in the oocyte and/or a failure to maintain the imprint in preimplantation development. In agreement, relaxed MEST imprinting was observed in both IVF and ICSI-derived embryos from couples presenting with different causes of infertility, and therefore can occur independently of an effect associated exclusively with male infertility (Table 1). We note that relaxed MEST imprinting was observed in embryos that were graded as 1 and 2, therefore implying that embryo morphological grade is not a useful indicator for the assessment of embryo epigenetic status, at least for MEST. Two sibling IVF blastocysts (sample 2, Figure 2a and sample
11, Figure 3) demonstrated contrasting states for MEST imprinting, with monoallelic and predominantly monoallelic expression, respectively. As these sibling embryos were fertilised and cultured together, it is not likely that the culture environment or an effect associated with female infertility is the causative factor in the embryo with relaxed imprinting; however, developmental differences between the embryos must be considered. Other possible causes of variability in MEST imprinting between embryos may be differing responses to the superovulation regimen. ${ }^{19}$ Other studies have reported lower than average methylation of the MEST gene in children conceived through ART. ${ }^{20}$ These observations concur with our findings reported here and could conceivably result in a loss of MEST imprinting in the early embryo. Our study cohort included embryos that were fertilised in two different fertility clinics, suggesting that variable MEST imprinting may be common in in vitro-derived embryos, but may also occur in vivo. Due to insufficient material (genomic DNA), we were unable to establish whether LOI was present at other loci, that would imply a global imprinting defect. Development up to the blastocyst stage can however clearly be achieved in the embryos with relaxed MEST imprinting.

These concerns must however be balanced against current knowledge of the imprinting status of isoforms that arise from the MEST locus. Isoform 1 appears to be expressed in a monoallelic fashion in numerous tissues. ${ }^{7}$ Initial reports described isoform 2 as being biallelic in blood lymphocytes. ${ }^{41}$ Subsequent reports have indicated that in certain tissues including breast, placenta and colon, isoform 2 may in fact be imprinted and, moreover, that the imprinting status (whether monoallelic or biallelic) may differ between individuals. ${ }^{13,16,37,47}$ Isoform 2 has been observed to be preferentially paternally expressed in foetal placenta in other studies, and also in kidney and fibroblast lines, with polymorphic imprinting observed between different fibroblast lines. ${ }^{48}$ Variable imprinting of MEST, including that of isoform 2, has also been reported between humanembryonic stem cell (hESCs) lines, raising concerns of the epigenetic stability of these cells. ${ }^{40,49,50}$

In view of the observations of aberrant MEST methylation and imprinting with disease, ${ }^{13,14,16}$ we considered it important to further characterise expression at the MEST locus in the embryos with expression arising from both alleles. Our data demonstrates that isoform 2 was the predominant MEST isoform detected in preimplantation embryos and show directly that imprinting of isoform 2 itself is variable between embryos (Figure $4 \mathrm{~d}$ ). We were, however, unable to test biallelic embryos in the isoform 2-imprinting assay. We acknowledge that technical restrictions, or differential polyA tail length usage between isoforms, may have limited our ability to detect isoform 1 expression in oocytes and embryos (Figures $4 \mathrm{~b}$ and c, respectively). Significantly, we found no evidence for MEST imprinting states that are observed in disease, that is, either relaxation of imprinting of isoform 1, or of promoter shifting from imprinted isoform 1 to the variably imprinted isoform 2 . The variability in MEST imprinting between hESC lines and also the placenta may therefore reflect an epigenetic programme that was intrinsic to the founder blastocyst, within the inner cell mass and trophectoderm, respectively. We detected expression of the P1b MEST splice variant in a small number of oocytes (Figure $4 \mathrm{~b}$ ) and this is in agreement with the observation of expression of P1b in the ovary. ${ }^{42}$

In conclusion, while infertility or ART-induced effects both remain as possible causes for the variable imprinting of MEST observed among the large cohort of blastocysts tested, our considered opinion is that effects due to inter-individual variation in imprinting of isoform 2 must also be considered. These MEST imprinting 
differences may also exist in naturally conceived human preimplantation embryos. Regardless of the cause, the developmental consequences of the variable imprinting of MEST in early human embryos in vitro and in vivo remain unclear. In mice, elevated expression of Mest has been associated with fat mass expansion 4,51,52 and obese phenotypes with LOI of Mest have been obtained from epigenetically immature oocytes. ${ }^{53}$ Finally, it remains possible that uncharacterized MEST transcripts, such as human equivalents of the longer MestXL variants that were recently described in the mouse, ${ }^{54}$ may have been amplified in our non-isoform-specific PCR assays. These observations suggest that further characterisation of the causes and effects of contrasting embryonic imprinting states during in vitro conception are required.

\section{CONFLICT OF INTEREST}

The authors declare no conflict of interest.

\section{ACKNOWLEDGEMENTS}

This work was supported by the Newlife Foundation and the MRC (Grant no. G0701388). We thank Judith A Hawkhead (University of York) for assistance with sample collection.

1 Beechey CV: Peg1/Mest locates distal to the currently defined imprinting region on mouse proximal chromosome 6 and identifies a new imprinting region affecting growth. Cytogenet Cell Genet 2000; 90: 309-314.

2 Lefebvre L, Viville S, Barton SC, Ishino F, Keverne EB, Surani MA: Abnormal maternal behaviour and growth retardation associated with loss of the imprinted gene Mest. Nat Genet 1998; 20: 163-169.

3 Takahashi M, Kamei Y, Ezaki O: Mest/Peg1 imprinted gene enlarges adipocytes and is a marker of adipocyte size. Am J Physiol Endocrinol Metab 2005; 288: E117-E124.

4 Kamei Y, Suganami T, Kohda T et al: Peg1/Mest in obese adipose tissue is expressed from the paternal allele in an isoform-specific manner. FEBS Lett 2007; 581: 91-96.

5 Nikonova L, Koza RA, Mendoza T, Chao PM, Curley JP, Kozak LP: Mesoderm-specific transcript is associated with fat mass expansion in response to a positive energy balance. FASEB J 2008; 22: 3925-3937.

6 Jung $\mathrm{H}$, Lee SK, Jho EH: Mest/Peg1 inhibits Wnt signaling via regulation of LRP6 glycosylation. Biochem J 2011; 436: 263-269.

7 Kobayashi S, Kohda T, Miyoshi $\mathrm{N}$ et al: Human PEG1/MEST, an imprinted gene on chromosome 7. Hum Mol Genet 1997; 6: 781-786.

8 Riesewijk AM, Hu L, Schulz U et al: Monoallelic expression of human PEG1/MEST is paralleled by parent-specific methylation in fetuses. Genomics 1997; 42: 236-244.

9 Riesewijk AM, Blagitko N, Schinzel AA et al: Evidence against a major role of PEG1/ MEST in Silver-Russell syndrome. Eur. J Hum Genet 1998; 6: 114-120.

10 Kobayashi S, Uemura H, Kohda T et al: No evidence of PEG1/MEST gene mutations in Silver-Russell syndrome patients. Am J Med Genet 2001; 104: 225-231.

11 Schöherr N, Jäger S, Ranke MB et al: No evidence for isolated imprinting mutations in the PEG1/MEST locus in Silver-Russell patients. Eur J Med Genet 2008; 51 322-324.

12 Shi W, Lefebvre L, Yu Y et al: Loss-of-imprinting of Peg1 in mouse interspecies hybrids is correlated with altered growth. Genesis 2004; 39: 65-72.

13 Nishihara S, Hayashida T, Mitsuya $\mathrm{K}$ et al: Multipoint imprinting analysis in sporadic colorectal cancers with and without microsatellite instability. Int J Oncol 2000; 17: 317-322.

14 Nakanishi $\mathrm{H}$, Suda $\mathrm{T}$, Katoh $\mathrm{M}$ et al: Loss of imprinting of PEG1/MEST in lung cancer cell lines. Oncol Rep 2004; 12: 1273-1278.

15 Pedersen IS, Dervan PA, Broderick D et al: Frequent loss of imprinting of PEG1/MEST in invasive breast cancer. Cancer Res 1999; 59: 5449-5451.

16 Pedersen IS, Dervan P, McGoldrick A et al: Promoter switch: a novel mechanism causing biallelic PEG1/MEST expression in invasive breast cancer. Hum Mol Genet 2002; 11: 1449-1453.

17 Huntriss J, Picton HM: Epigenetic consequences of assisted reproduction and infertility on the human preimplantation embryo. Hum Fertil (Camb) 2008; 11: 85-94.

18 Kagami M, Nagai T, Fukami M, Yamazawa K, Ogata T: Silver-Russell syndrome in a girl born after in vitro fertilization: partial hypermethylation at the differentially methylated region of PEG1/MEST. J Assist Reprod Genet 2007; 24: 131-136.

19 Sato $A$, Otsu $E$, Negishi $H$, Utsunomiya T, Arima T: Aberrant DNA methylation of imprinted loci in superovulated oocytes. Hum Reprod 2007; 22: 26-35.

20 Katari S, Turan N, Bibikova M et al: DNA methylation and gene expression differences in children conceived in vitro or in vivo. Hum Mol Genet 2009; 18: 3769-3778.

21 Tierling S, Souren NY, Gries J et al: Assisted reproductive technologies do not enhance the variability of DNA methylation imprints in human. J Med Genet 2010; 47: 371-376.
22 Imamura T, Kerjean A, Heams T, Kupiec JJ, Thenevin C, Paldi A: Dynamic CpG and non-CpG methylation of the Peg1/Mest gene in the mouse oocyte and preimplantation embryo. J Biol Chem 2005; 280: 20171-20175.

23 Anckaert E, Romero S, Adriaenssens T, Smitz J: Effects of low methyl donor levels in culture medium during mouse follicle culture on oocyte imprinting establishment. Biol Reprod 2010; 83: 377-386.

24 Kerjean $\mathrm{A}$, Couvert $\mathrm{P}$, Heams T et al: In vitro follicular growth affects oocyte imprinting establishment in mice. Eur. J Hum Genet 2003; 11: 493-496.

25 Lee Y,S, Latham KE, Vandevoort CA: Effects of in vitro maturation on gene expression in rhesus monkey oocytes. Physiol Genomics 2008; 35: 145-158.

26 Marques CJ, Costa P, Vaz B et al: Abnormal methylation of imprinted genes in human sperm is associated with oligozoospermia. Mol Hum Reprod 2008; 14: 67-74.

27 Hammoud SS, Purwar J, Pflueger C, Cairns BR, Carrell DT: Alterations in sperm DNA methylation patterns at imprinted loci in two classes of infertility. Fertil Steril 2010; 94: 1728-1733

28 Poplinski A, Tüttelmann F, Kanber D, Horsthemke B, Gromoll J: Idiopathic male infertility is strongly associated with aberrant methylation of MEST and IGF2/H19 ICR1. Int J Androl 2010; 33: 642-649.

29 Kobayashi H, Sato A, Otsu E et al: Aberrant DNA methylation of imprinted loci in sperm from oligospermic patients. Hum Mol Genet 2007; 16: 2542-2551.

30 Houshdaran S, Cortessis VK, Siegmund K, Yang A, Laird PW, Sokol RZ: Widespread epigenetic abnormalities suggest a broad DNA methylation erasure defect in abnormal human sperm. PLoS One 2007; 2: e1289.

31 Huntriss J, Woodfine K, Huddleston JE et al: Quantitative analysis of DNA methylation of imprinted genes in single human blastocysts by pyrosequencing. Fertil Steril 2011; 95: 2564-2567.

32 Houghton FD, Hawkhead JA, Humpherson PG et al: Non-invasive amino acid turnover predicts human embryo developmental capacity. Hum Reprod 2002; 17: 999-1005.

33 Ghassemifar MR, Eckert JJ, Houghton FD, Picton HM, Leese HJ, Fleming TP: Gene expression regulating epithelial intercellular junction biogenesis during human blastocyst development in vitro. Mol Hum Reprod 2003; 9: 245-252.

34 Tay JI, Rutherford AJ, Killick SR, Maguiness SD, Partridge RJ, Leese HJ: Human tubal fluid: production, nutrient composition and response to adrenergic agents. Hum Reprod 1997; 12: 2451-2456.

35 Eberwine J, Yeh $\mathrm{H}$, Miyashiro $\mathrm{K}$ et al: Analysis of gene expression in single live neurons. Proc Natl Acad Sci USA 1992; 89: 3010-3014.

36 Wang E, Miller LD, Ohnmacht GA, Liu ET, Marincola FM: High-fidelity mRNA amplification for gene profiling. Nat Biotechnol 2000; 18: 457-459.

37 McMinn J, Wei M, Sadovsky Y, Thaker HM, Tycko B: Imprinting of PEG1/MEST isoform 2 in human placenta. Placenta 2006; 27: 119-126.

38 Salpekar A, Huntriss J, Bolton V, Monk M: The use of amplified cDNA to investigate the expression of seven imprinted genes in human oocytes and preimplantation embryos. Mol Hum Reprod 2001; 7: 839-844.

39 Cui H, Horon IL, Ohlsson R, Hamilton SR, Feinberg AP: Loss of imprinting in norma tissue of colorectal cancer patients with microsatellite instability. Nat Med 1998; 4: $1276-1280$

40 Kim KP, Thurston A, Mummery C et al: Gene-specific vulnerability to imprinting variability in human embryonic stem cell lines. Genome Res 2007; 17: 1731-1742.

41 Kosaki K, Kosaki R, Craigen WJ, Matsuo N: Isoform-specific imprinting of the human PEG1/MEST gene. Am J Hum Genet 2000; 66: 309-312.

$42 \mathrm{Li} \mathrm{T}, \mathrm{Vu} \mathrm{TH}$, Lee $\mathrm{KO}$ et al: An imprinted PEG1/MEST antisense expressed predominantly in human testis and in mature spermatozoa. J Biol Chem 2002; 277: $13518-13527$

43 Lighten AD, Hardy K, Winston RM, Moore GE: IGF2 is parentally imprinted in human preimplantation embryos. Nat Genet 1992; 15: 122-123.

44 Huntriss J, Daniels R, Bolton V, Monk M: Imprinted expression of SNRPN in human preimplantation embryos. Am J Hum Genet 1998; 63: 1009-1014.

45 Monk M, Salpekar A: Expression of imprinted genes in human preimplantation development. Mol Cell Endocrinol 2001; 183:Suppl 1 S35-S40.

46 McElroy SL, Byrne JA, Chavez SL et al: Parthenogenic blastocysts derived from cumulus-free in vitro matured human oocytes. PLoS One 2010; 5: e10979.

47 Diplas Al, Lambertini L, Lee MJ et al: Differential expression of imprinted genes in normal and IUGR human placentas. Epigenetics 2009; 4: 235-240.

48 Nakabayashi K, Bentley L, Hitchins MP et al: Identification and characterization of an imprinted antisense RNA (MESTIT1) in the human MEST locus on chromosome 7q32. Hum Mol Genet 2002; 11: 1743-1756.

49 Adewumi O, Aflatoonian B, Ahrlund-Richter L et al: Characterization of human embryonic stem cell lines by the International Stem Cell Initiative. Nat Biotechnol 2007; 25: 803-816.

50 Frost JM, Monk D, Moschidou D et al: The effects of culture on genomic imprinting profiles in human embryonic and fetal mesenchymal stem cells. Epigenetics 2011; 6: $52-62$.

51 Koza RA, Rogers P, Kozak LP: Inter-individual variation of dietary fat-induced mesoderm specific transcript in adipose tissue within inbred mice is not caused by altered promoter methylation. Epigenetics 2009; 4: 512-518.

52 Koza RA, Nikonova L, Hogan J et al: Changes in gene expression foreshadow dietinduced obesity in genetically identical mice. PLoS Genet 2006; 2: e81.

53 Obata Y, Hiura H, Fukuda A, Komiyama J, Hatada I, Kono T: Epigenetically immature oocytes lead to loss of imprinting during embryogenesis. J Reprod Dev 2011; 57: 327-334

54 Macisaac J, Bogutz A, Morrissy A, Lefebvre L: Tissue-specific alternative polyadenylation at the imprinted gene Mest regulates allelic usage at Copg2. Nucleic Acids Res 2012; 40: 1523-1535. 